\title{
Comments on SIC design for SISO NOMA Systems over Doubly Selective Channels.
}

This paper was downloaded from TechRxiv (https://www.techrxiv.org).

\section{LICENSE}

CC BY 4.0

SUBMISSION DATE / POSTED DATE

$15-02-2022$ / 22-02-2022

\section{CITATION}

Kontorovich, Valeri (2022): Comments on SIC design for SISO NOMA Systems over Doubly Selective Channels.. TechRxiv. Preprint. https://doi.org/10.36227/techrxiv.19178423.v1

$\mathrm{DOI}$ 


\title{
Comments on SIC design for SISO NOMA Systems over Doubly Selective Channels.
}

\author{
V. Kontorovich, Life Fellow, IEEE
}

\begin{abstract}
Non-orthogonal multiple access principle applied in power domain (power-domain NOMA) enhances spectrum efficiency and connectivity of mobile communication systems through sharing between Orthogonal Multiple Access (OMA) and other multiple users. It is the almost main current trend for research and standards for $5 \mathrm{G}$ and beyond. Spectrum sharing generates multi-users interference (Multiple Access Interference, MAI) which destroys orthogonality in OMA systems. Meanwhile the so-called Successive Interference Cancellation (SIC) techniques are used to suppress MAI and the Cognitive Radio (CR) principle (CR-NOMA) for decoding order for multiple users inspired effective SIC algorithms. The CR-NOMA principle is applied in the present material as well, but with significant changes for SIC design as for Doubly Selective channels. Due to the above reasons, two original SIC design methods are proposed hereafter following the CR-NOMA principle. Regarding to it the OMA signal (primary user) is decoded first using a Chaos-based quasi-optimum Extended Kalman Filter (EKF). The set of "secondary users" (multiusers) are proposed to be decoded secondly by the following two different methods:

- Chaos-based EKF filters or ,

- Sequential $m$ hypothesis testing.

The proposed approach for SIC design is rather simple, fast and accurate for practical implementation.
\end{abstract}

Index Terms-Doubly Selective Channels, Interference Cancelation, Non-Linear Filtering, Orthogonal Access.

\section{INTRODUCTION ${ }^{1}$}

$\mathrm{N}$ ON-orthogonal Multiple Access (NOMA) is a perspective implementation paradigm for $\mathbf{5 G}$ and beyond mobile communication standards and systems (both for uplink and downlink) in order to improve mobility, connectivity and spectrum efficiency due to the constantly growing demand.

One of the main problems that emerge in this regard is how to mitigate excessive interferences caused by the spectrum sharing between multiusers and conventional OMA. To significantly reduce such kind of Multiple Access Interferences (MAI) the so-called SIC (Successive Interference Cancellation) approach was recommended (see [1]-[3] and references therein). Although the successive cancellation method for interference mitigation was, in general, well known for many years, the SIC approach has been recently at the center of the attention for both the research community and the industry professionals. Numerous original proposals have been published in order to modify and improve the basic principle of the sequential

Paper submitted on Jan. ???, 2022. "This work was partially supported by the Intel Co under Grant "Intel-VK".

V.Kontorovich is with the Electrical Engineering Department(Communication Section), at the Research and Advanced Studies Center (CINVESTAV), Mexico City, CP 07360 (e-mail: valeri@cinvestav.mx). nature of SIC applying features and characteristics such as Channel State Information (CSI), details of system behavior, etc. (see details at [1], [3], etc.). A brief overview of the works considered as most important [1-12] is presented in the following.

As it can be inferred from [4]-[5], the SIC decoding order based on the CSI turns critical and can have important influence on the system's performance.

Authors of [6] and [8] suggest the usage of combined (OMA/NOMA). In [6] partial-NOMA is introduced in a large two-user downlink network in order to provide throughput and reliability. The associated partial overlap controls interference while still offering spectrum reuse. The nature of the partial overlap also allows to employ receive-filtering to further suppress interference. For signal decoding a flexible successive interference cancellation (FSIC) decoding was proposed and compared with OMA and NOMA performance.

In [6] a problem to maximize the total throughput constrained to a minimum throughput requirement for each user was formulated and an algorithm to efficiently find a feasible resource allocation was proposed. The results show that partial-NOMA allows greater flexibility in terms of performance. Partial-NOMA can also serve users that NOMA cannot. Different from the proposal made in this work, NOMA users in [6] cannot support high transmission rate requirements.

In [8] an iterative SIC receiver architecture with pilot- 
and data-based channel estimation was proposed for efficient decoding of non-orthogonal superimposed signals. It was claimed that non-orthogonal superposition concept on top of OFDMA is a promising technique to improve cell spectral efficiency. In the cellular case, the SIC multi-user receiver scheme is well adapted for user signal separation. However, authors in [8] do not work with high-mobility scenarios that entail Doubly Selective Channels.

Notice, that almost every proposed SIC technique in the literature strongly depends on the CSI [12]. However, the usage of the Channel State Information (CSI) or its modifications [1], [2], [6], [10] is hardly possible, due to the Doubly Selective Channels. Contrary to the previous published papers, new findings reported in this work consider the SIC impairments mitigation related to double selectivity troubles.

The SIC methods for SISO channels have been extensively studied and the results are reported in the literature. Nevertheless, 5G and beyond networks have proposed also MIMO techniques in order to increase capacity. Furthermore, with the employment of Massive MIMO techniques the usage of CSI becomes challenging since the number of users goes large [6]-[12]. In [6] a blind belief propagation (BP) detection was proposed for noncoherent NOMA with massive MIMO, where the transmitter of each user first performs differential modulation on PSK symbols, and then spreads its symbols using low-density spreading (LDS); the receiver of the base station (BS) employs differential demodulation and then detects all users' symbols using a blind BP detection without knowledge of CSI. Strictly speaking this approach is not related to power domain NOMA, it rather belongs to CDMA-NOMA, but it was included here because the idea of applying incoherent transmission is rarely considered in the NOMA design.

The new findings proposed in this paper show that the use of CR-NOMA principles could be successfully applied to separate users in different scenarios without any Channel State Information, corresponding precisely to Incoherent ideology of NOMA transmission.

Once more it reasonable to stress: careful analysis of the existing SIC proposals shows that when one wishes to apply any of them particularly to the rather attractive CRNOMA, and its modifications) for NOMA design over Doubly Selective channels for information transmission from High Speed Vehicles (HSV) some significant changes and improvements are required.

It is time to mention, that Doubly Selective Channel is the typical scenario for information transmission from High Speed Vehicles (HSV) such as trains, cars, aircrafts, etc. (see, for example, the case of uplink communication from HSV to the nearest base station and so on).

As it was shown in [13]-[16] the transmission systems design over Doubly Selective channels needs to take into account several main features of such channels:

- Strongly selective and severe (deep) fading in time and frequency domains.
- Relatively large values of Doppler shifts and Frequency Offsets.

- Large range of average Signal to Noise Ratio (SNR) variations at the receiving terminals for all the users, etc.

In order to overcome those harmful channel impairments, effective principles for the system design were proposed earlier (see [13]-[16] and the references therein) based on the invariant DPSK-k modulation, virtual trajectories method for channel orthogonal decomposition, incoherent demodulation algorithms, etc.

Therefore, the main part of the CR-NOMA proposals cited above might be hardly possible to adjust to Doubly Selective channels, for the same reasons that were stated and discussed in [13], [14], so the SIC design approach needs to be certainly modified. The principles and possible realizations for this SIC will be addressed in the following material.

It is opportune to stress here that one of the main limitations of the power-domain NOMA is, that in order to achieve reasonable performance gain over OMA, the channel conditions for users need to be sufficiently different. The concrete meaning of this will be illustrated (numerically) in the following. Related practical scenarios of spectrum sharing for completely different services were already discussed in [1], [3], etc; note, that mainly they are considered hereafter as well.

Taking the latter into account, the main idea of the CRNOMA, i.e., the OMA (primary signal) is to be decoded first by the Filter for OMA (FOMA) and then the rest of the multiusers (secondary users) go for decoding, is introduced here but with significant differences in the essence and content for the SIC processing.

For the SIC decoding order of the multi-users two different approaches are proposed, namely:

- A Chaos- based filtering bank method and

- Multiple sequential hypothesis testing procedure.

It is worth mentioning here that according to the references (see [1], [3]) and our own study (see below), the OMA signal and the multiuser signals can be successfully approximated by means of a Gaussian random process model. Another reason for the Gaussian approximation of the desired signals at the SIC input is that, Doubly Selective Channels due to their physical nature (high selectivity in both domains) "shift" signals (no matter what service they belong to) to an "almost" Gaussian random process. The latter gives additional "benefits" for the Chaos-based filtering approach [17]-[20] broadly applied at the blockdiagrams of figures 1 and $2^{2}$ which will be thoroughly explained in the following section.

For the OMA decoding in both options a Chaos -based filtering method was chosen. Thus, the idea of SIC processing algorithms can be illustrated in figure 1 and figure 2 .

\footnotetext{
${ }^{2}$ Note that the elements of the block-diagrams in figures 1 and 2 can be considered as located in space as they might belong to different services [3].
} 


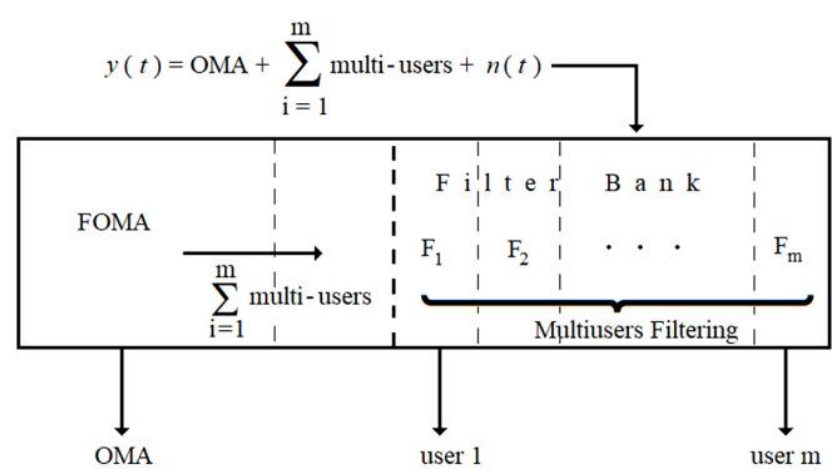

Figure 1. SIC processing (classifier) block with application of Filtering approach.

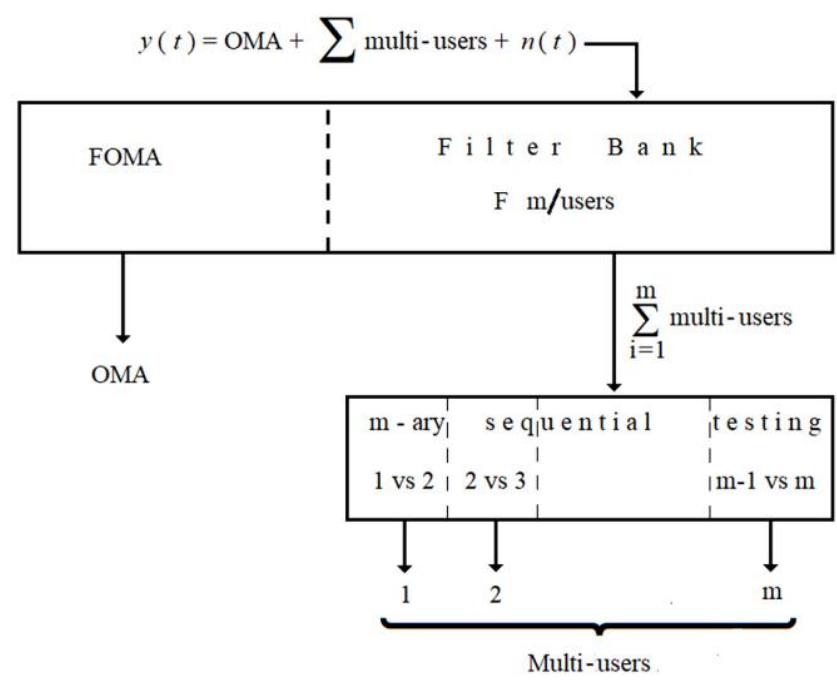

Figure 2. SIC processing (classifier) block with application of Sequential Hypothesis Testing approach.

It can be seen, that the SIC processing is nothing else, but a pre-processing before the appropriate demodulation of all the desired signals or services (OMA and multiusers). This pre-processing SIC device is actually not decoding,but classifying the OMA signal among the multiuser signals and then each multiuser among themselves. Thus, it is important to stress here that the evaluation metrics applied hereafter need to be chosen in the way to be adequate for classification issues (see section IV). All the details for each of the elements of the block-diagrams in figure 1 and figure 2 will be presented in the coming sections.

In order to avoid any misleading hereafter it is reasonable to stress, that in Figures 1 and 2 the $y(t)$ represents the baseband aggregated signal at the SIC input!

The paper is organized as follows. Section II is completely dedicated to Chaos-based filtering algorithms applied both to the OMA signal and to the set of multiuser interferers. Section III illustrates the principles of filtering of the OMA signals in presence of multiusers interference and Additive Gaussian Noise (AWGN) together with principles of multiusers filtering as well. The main attention will be stressed on the high precision of the Chaos-based filtering for decoding of the OMA signal and the rest of the multi-users by means of simulation results. Section IV is devoted to the Sequential Analysis Testing of $m$ hypotheses for multiusers. For the hypotheses, each signal of the multiusers will be considered in the form of the time set of samples of a Gaussian process with means and variances different for each of the multiusers.

\section{SKetch Of The ChaOs-BASed FILTERING ALGORITHMS.}

\section{A. Generalities}

Chaotic signals as models for real physical phenomena are well known during the last decades (see [17]-[21] and the fundamental references therein). Particularly for the purpose of interference mitigation they were applied rather recently and showed very attractive characteristics regarding filtering precision and efficient interference mitigation.

As an example, one can consider the processing algorithms based on chaos filtering for mitigation of Radio Frequency Interference (RFI) produced by desktops and laptops [21], [22]. In this regard the attempt to implement the Chaos-based filtering for OMA and multiusers decoding looks almost natural.

So, what is the idea? Continuous chaotic signals, applied hereafter, are generated by deterministic nonlinear systems (strange attractors) and are described generally by Ordinary Differential Equations (ODE) of the type:

$$
\dot{\boldsymbol{x}}=\boldsymbol{F}(\boldsymbol{x}, t),
$$

where $\boldsymbol{x}$ is an n-dimensional vector of the attractors output signals, $\boldsymbol{F}(\cdot)$ is a known n-dimensional vector function and $\boldsymbol{x}\left(\boldsymbol{t}_{\mathbf{0}}\right)=\boldsymbol{x}_{0}$ are initial conditions.

In the following three concrete types of attractors will be applied: Lorenz, Chua and Rossler; their statistical features are presented in [21]. Thanks to the fundamental ideas of A. Kolmogorov and M. Born (see references in [17], [20], [21]) the statistical characterization of the deterministic systems (1) is well established by introducing at (1) a small so-called white noise process (process noises), which converts the ordinary equation (1) into stochastic differential equation (SDE) with very "small" elements of diffusion matrix.

Special cases for (1) applied hereafter (in discrete time) are:

Rossler attractor

$$
\begin{aligned}
& x_{k+1}=x_{k}+T_{s}\left(-y_{k}-z_{k}\right) \\
& y_{k+1}=y_{k}+T_{s}\left(x_{k}+0.2 y_{k}\right) \\
& z_{k+1}=z_{k}+T_{s}\left(0.2-z_{k}\left(5.7-x_{k}\right)\right)
\end{aligned}
$$

Lorenz attractor

$$
\begin{aligned}
& x_{k+1}=x_{k}+T_{s}\left(10\left(x_{k}-y_{k}\right)\right) \\
& y_{k+1}=y_{k}+T_{s}\left(28 x_{k}-y_{k}+x_{k} z_{k}\right) \\
& z_{k+1}=z_{k}+T_{s}\left(x_{k} y_{k}-\frac{8}{3} z_{k}\right)
\end{aligned}
$$


Chua attractor

$$
\begin{aligned}
& x_{k+1}=x_{k}+T_{s}\left(9.205\left(y_{k}-V\left(x_{k}\right)\right)\right) \\
& y_{k+1}=y_{k}+T_{s}\left(x_{k}-y_{k}+z_{k}\right) \\
& z_{k+1}=z_{k}-T_{s}\left(14.3 y_{k}\right)
\end{aligned}
$$

Where: $\quad V\left(x_{k}\right)=m_{1} x_{k}+\frac{1}{2}\left(m_{0}-m_{1}\right)\left[\left|x_{k}+1\right|-\left|x_{k}-1\right|\right]$; $m_{0}=-\frac{1}{7}, m_{0}=\frac{2}{7}$. At (2)-(4) $T_{s}$ is the sampling time.

Any component or all of them taken together from equations (2)-(4) might be considered as a model of the real physical signal, but in the following only one component from (2)-(4) will be applied as a model, which is denoted as the observable component.

Currently the theory and practice of the Chaos-based filtering algorithms for different signals are exhaustively described in [20], [21], and so there is no sense to repeat it here. Because of this, in the following only the concrete filtering algorithms for attractors (2)-(4) will be presented.

The latter was thoroughly investigated at [17], [20]-[22] and the extraction of the obtained results is as follows:

- With the balance between the filtering accuracy and the computational complexity, as an "optimum" option the Extended Kalman Filter (EKF) can be chosen, implemented in the so-called "one-moment" (1MM EKF) or two-moment (2MM EKF) time fashion.

- Taking into account that both the OMA and the set of multiusers can be well approximated via Gaussian models (see also Section III) the Standard (optimum) Kalman Filter (SKF) can be applied as well, but the relative preference of the EKF for some scenarios will be explained below.

Although there is possible to arise some "controversy" here, it is not the case: everything depends on the "double view" for the filtering problem formulation: on the nonlinearity of the Chaotic modeling of the signals on one side; and on the Gaussian approximation of its first order PDF on the other side. Note, that the optimality of SKF for strictly Gaussian stochastic processes described by its Gaussian $\mathrm{n}$-dimensional PDF, where $\mathrm{n} \rightarrow \infty$, or its Linear models is obviously out of question (details can be found, for example, in [18], [19])

- One-moment time instant (1MM) and two-moment time instants (2MM) algorithms (see [17], [20] and the references therein) differ in filtering accuracy, but they differ very lightly in terms of computational complexity and processing time, both being rather "fast". So, their implementation depends mainly on the concrete NOMA transmission system requirements.

- In general, an analytical comparison of the accuracy between the EKF and the SKF for all cases is difficult to calculate but taking into account that models (1)-(4) are deterministic and significantly nonlinear, the accuracy of the EKF might be better than the accuracy of SKF for this concrete cases. Reasons for this were "hinted" above and details can be found at [20].

\section{B. EKF (1MM and 2MM) Algorithms for Chaotic} Attractors (2)-(4)

The aim this sub-section is to present concrete algorithms of Chaos-based filtering which will be tested in the next section in order to choose the appropriate attractor model and the discrete time algorithm for filtering OMA and multiuser interferences at figures 1 and 2.

The theory of 1MM SKF and EKF is well known and developed in [18], [19]. Shortly, their aim is to "extract" the desired signal from the aggregated signal (with noise), whose model is known a priori and is used to properly "tune" the filter.

So, here only the correspondent final results will be reproduced with the corresponding explanations.

As it follows from [18]-[20] the SKF algorithm for the observable component (let it be " $x$ " at (1)-(4)) is optimum for the linear dynamic system in (1) and it consists of two cycles: prediction and correction:

$\begin{array}{ll}\text { Prediction } & \left\{\begin{array}{l}\hat{x}_{k+1}^{-}=f\left(\hat{x}_{k}^{+}\right) \\ P_{k}^{-}=A_{k} P_{k}^{+} A_{k}^{T}+Q_{k}\end{array}\right. \\ \text { Correction }\left\{\begin{array}{l}G_{k}=P_{k}^{-} H_{k}^{T}\left[H_{k} P_{k}^{-} H_{k}^{T}+N_{0_{k}}\right]^{-1} \\ \hat{x}_{k}^{+}=\hat{x}_{k}^{-}+G_{k}\left[y_{k}-s\left(x_{k}^{-}\right)\right] \\ P_{k}^{+}=P_{k}^{-}+G_{k} H_{k} P_{k}^{-}\end{array}\right.\end{array}$

The meanings of all parameters at (5), (6) will be explained a bit later.

The algorithm for EKF 1MM is the same as (5) and (6) but with a small modification due to its quasi-linearity, as shown in the following. Thus, it has to be once more stressed that the SKF is an optimum algorithm for the strongly Gaussian signals and their linear models and the $1 \mathrm{MM}$ EKF algorithm is quasi-optimum for nonlinear Chaos models of the (2)-(4) type, so the accuracy of its filtering (in our case in terms of Normalized Mean Squared errorNMSE) strictly speaking might be not the highest one, as it is shown in [20], but anyway it is practically acceptable for the majority of applications.

The 2MM EKF algorithm was proposed recently [17], [20] and is totally heuristic; besides it is quasi-optimum as well. The accuracy of the 1MM EKF and 2MM EKF does not demonstrate completely their "singular" properties as optimum ones (see, [17], [20]), but anyway those algorithms are accurate, highly efficient and attractive for applications.

In general, one has to notice (see for theory [17], [20]), that for all attractors (2)-(4) the statistical system dynamics and the measurement of the state of the system are tied to the statistically independent so-called "process noises"(the reason for their introduction was mentioned above) with the a priori known rather small elements of their covariance 
matrixes given by $N_{0_{k}}$ and $Q_{k}{ }^{3}$ respectively(see also Tables1,2 in the following) and s(.) is a general form for the desired(filtered) signal. Note, that in further concrete applications the form $\mathrm{s}(\mathrm{)})$ is omitted.

It is worth to mention here that the latter explains, how the correction term in filtering algorithms of (6) type is related to the very small intensities of the process noise and therefore has a rather small or negligible influence on the Chaos filtering process, leaving "almost" only the prediction part and providing finally "singular" properties for filtering accuracy (see below as well).

One has to notice as well, that as all the models (1)-(4) are presented in the form of the Differential Equations or Difference Equations, their filtering algorithms are of recurrent type.

Now it is time to define all the parameters at (5), (6): $G_{k}$ is the so-called Kalman gain, $\hat{x}_{k}^{-}$is the a priori state estimate in the $k$-th update cycle, $\hat{x}_{k}^{+}$is the a posteriori state estimate in the $k$-th update cycle, $P_{k}^{-}, P_{k}^{+}$are respectively the a priori and the a posteriori error covariance matrix estimates in the k-th cycle, $A_{K}$ is the fix state transition Matrix (for SKF) or for the 1MM EKF the linearization matrix (Jacobian) $A_{k}=\frac{\partial f\left(x_{k}\right)}{\partial x_{k}}$ which denotes "quasi-linearity" for the 1MM EKF. For the 2MM EKF (see [21]) in (5):

$$
P_{k}^{-}=A_{k} P_{k}^{+}\left(1-\rho^{2}\right) A_{k}^{T}+Q_{k}
$$

where $\rho$ is the correlation coefficient of two-moment adjacent samples. As it was stated at $/ 18,19 /$ it's value have to be chosen between $0,7-0,85$.

The rest of the equations (5) and (6) remain unchanged and it can be easily seen that the filtering algorithm in general is the same [17], [20]. Thus, the processing time (number of samples) for 1MM EKF and 2MM EKF are almost the same, but the filtering accuracy might differ. The latter was illustrated for signals of different physical nature in [17], [20], where the interested reader can find more details for the matter.

It is worth mentioning once more here that Chaos-based filtering algorithms are generally based on Ordinary Differential Equations (ODE) of chaotic deterministic dynamics and have a very important and spectacular property, the so-called "singularity" for their solutions. In other words, the filter's output (estimate) signal is somehow "tuned" to its a-priori dynamics (or to its mathematical model) applied in the filtering algorithm in the prediction term. That's why the filtering accuracy is high, even for quasi-optimum algorithms and the filtering fidelity (hereafter, in terms of the Normalized Mean Square Error,

\footnotetext{
${ }^{3}$ Introducing those noises according to A. Kolmogorov and M. Born is fundamental for statistical characterization of the deterministic systems (see references at [17], [20])
}

NMSE as a metric theoretically adequate for filtering accuracy [18], [19]) shows a rather low dependence to the input SNR, i.e. it demonstrates its singular features (see also [19]). The latter will be illustrated by means of simulation results in the next section as well.

\section{Simulation Results}

Simulations presented in this section are dedicated to explaining the Chaos-based filtering for the primary OMA signal which is modeled hereafter as an OFDMA signal in presence of NOMA multiuser signals and AWGN channel noise. Here, the sum of the multiusers is modeled as a sum of non-orthogonal (NOMA) carriers. Note that as a reference, the choice of the simulation parameters was made according to the IEEE802.11.a Standard, where the number of carriers (at least for OMA) is chosen around 50.and is applied in all simulation hereafter.

Next, the results of the NOMA filtering in presence of AWGN will be presented.

One has to notice that the scenario when the multiuser signals are filtered by the bank of the Chaos-based filters, where each element belongs to the specific multiuser, actually might be solved in the same way, assuming (as it is hereafter done) that the OMA signal is extracted from the input.

The way to simulate OFDMA and NOMA multicarrier signals is the same: by manipulating the carrier's central frequencies with constant integers (OFDMA case) or noninteger constants, say $\delta=1.2$, (NOMA case), similar to NOMA generation in the literature (see, [7]).

The set of corresponding signals were processed in order to obtain their statistical characteristics: PDF and autocorrelation. The results are illustrated in figures 3 and 4 that show that NOMA interference can be considered as Additive Colored Gaussian noise, ACGN, (similar models are applied in [1], [3], etc. and at the next section as well).
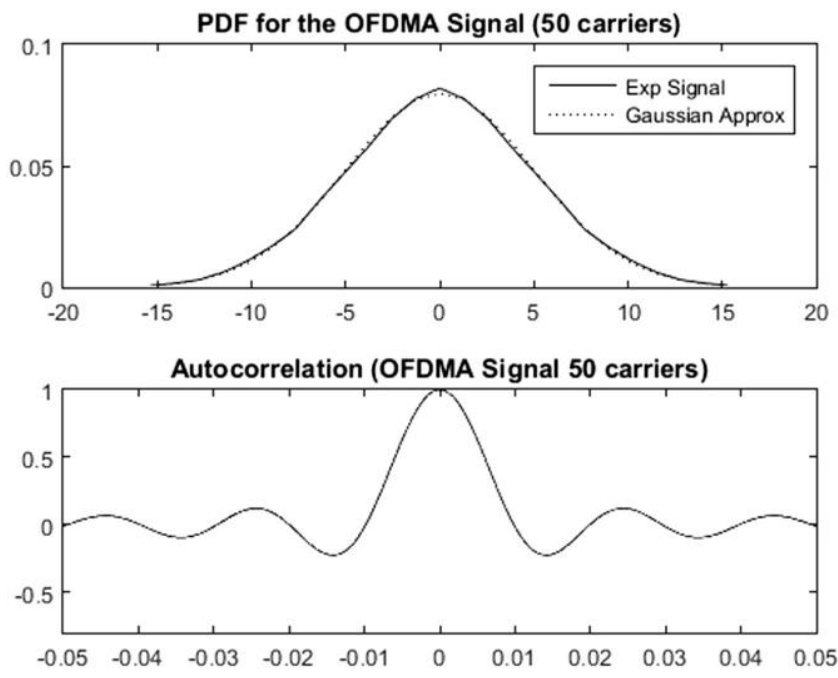

Figure 3. PDF and autocorrelation for the OFDMA signal. 

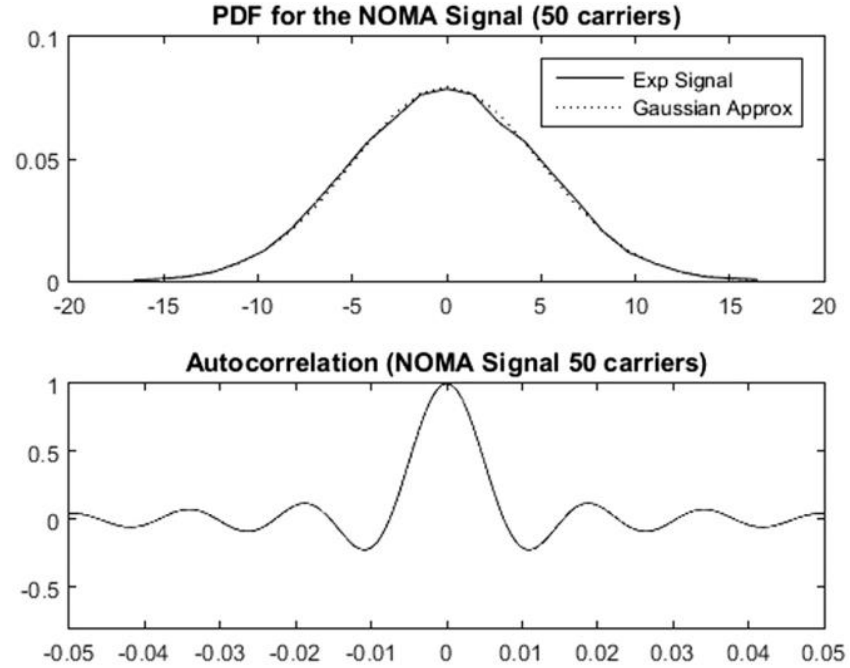

Figure 4. PDF and autocorrelation for the NOMA signal.

In the following we explore how effective the EKF based non-linear filtering (IMM and 2MM) can be, when it is applied to OFDMA (OMA) signals under the influence of AWGN and NOMA interference as shown in figure 5.

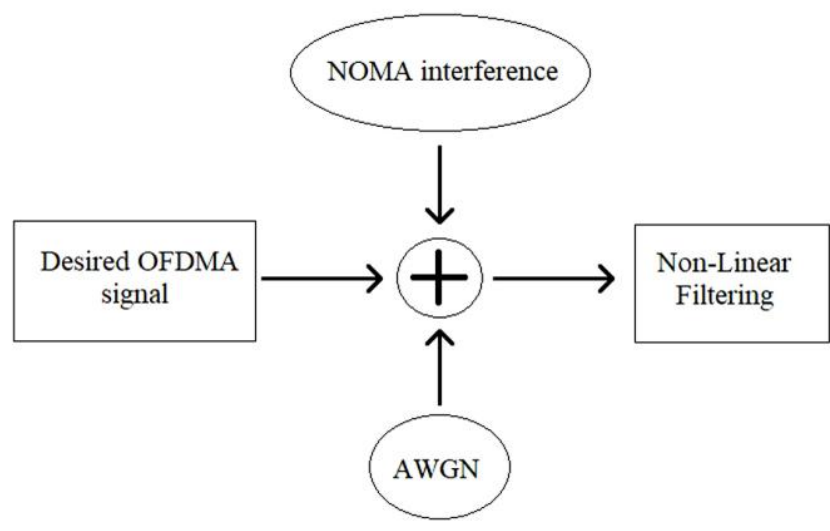

Figure 5. Filtering scenario.

The same block-diagram might be applied for investigation of NOMA filtering in presence of AWGN by eliminating the OFDMA generation at Fig.5.

First the results of filtering OFDMA in presence of NOMA interferences and AWGN are presented using EKF algorithm with $1 \mathrm{MM}$ and $2 \mathrm{MM}$ estimates for different signal to noise ratios (SNR), signal to interference ratios (SIR) and interference to noise ratios (INR). But previously it has to be verified what kind of attractors are statistically adequate for OFDMA and NOMA modeling. After testing the $x, y$ and $z$ components of the Rossler, Lorenz and Chua Attractors, it was found by comparing the correspondent statistical characteristics, that only the $x$ component of the Rossler and Chua attractors could be used for further modeling and filtering, where the $1 \mathrm{MM}$ and $2 \mathrm{MM}$ filtering were processed according to algorithms (5)-(7). The results are presented in figures 6 and 7 and in Table 1. The Q denotes the process noise value from formulas (5) and (7).

For the 2MM EKF filtering according to formula (7) the value for the correlation coefficient is $\rho=0.589$.

Table 1. NMSE with SNR fixed in $15 \mathrm{~dB}$.

\begin{tabular}{|c|c|c|c|}
\hline & $\mathrm{SIR}=15 \mathrm{~dB}$ & $\mathrm{SIR}=12 \mathrm{~dB}$ & $\mathrm{SIR}=5 \mathrm{~dB}$ \\
\hline \multicolumn{4}{|c|}{ EKF Rossler $\mathrm{X} \quad \mathrm{Q}=0.9$} \\
\hline $1 \mathrm{MM}$ & 0.030 & 0.045 & 0.16 \\
\hline $2 \mathrm{MM}$ & 0.028 & 0.042 & 0.15 \\
\hline \multicolumn{4}{|c|}{ EKF Chua X $\quad Q=0.5$} \\
\hline $1 \mathrm{MM}$ & 0.047 & 0.071 & 0.26 \\
\hline $2 \mathrm{MM}$ & 0.045 & 0.068 & 0.25 \\
\hline
\end{tabular}

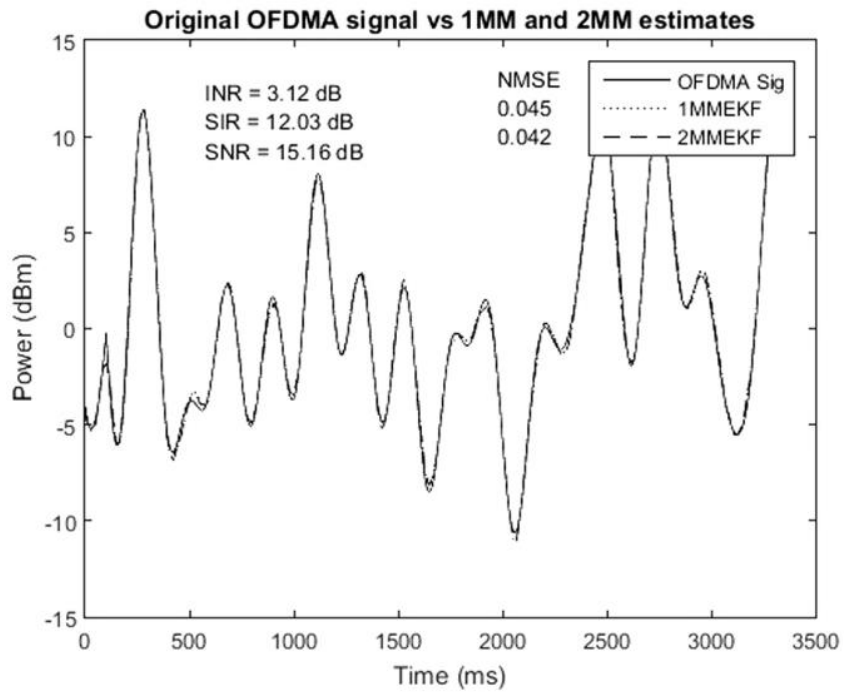

Figure 6. Signals for INR $=3 \mathrm{~dB}$ using Rossler $\mathrm{X}$.

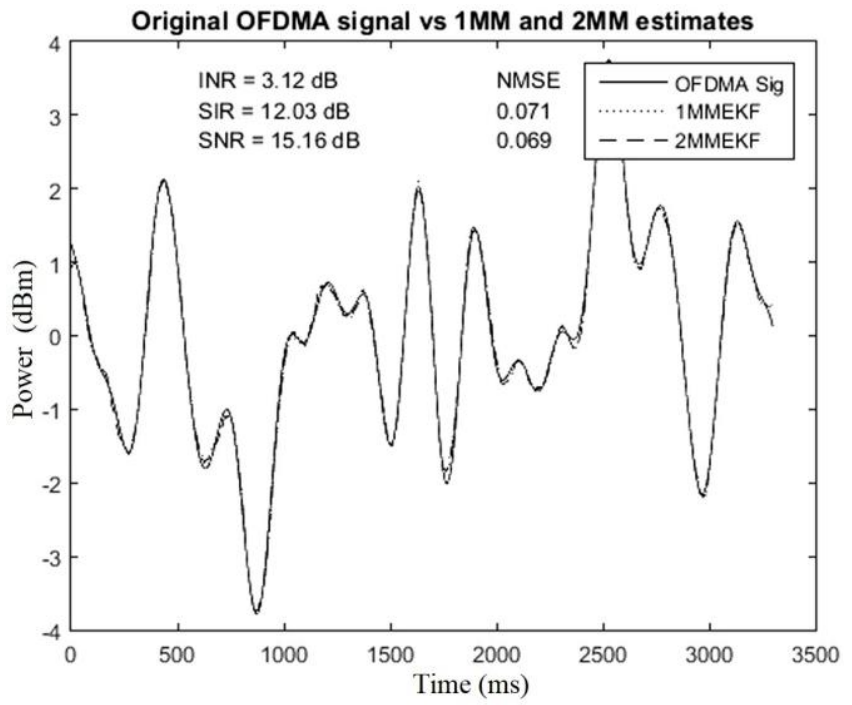

Figure 7. Signals for INR $=3 \mathrm{~dB}$ using Chua $\mathrm{X}$.

Here Rossler $\mathrm{X}$ and Chua $\mathrm{X}$ means $\mathrm{X}$-components for both attractors! It is worth to attract the attention to the results, presented in figures 6 and 7 and the table 1 .

It follows that for the SNR $=13-15 \mathrm{~dB}$ usually applied in wireless systems and SIR around the same order, the NMSE values are less than 5\%; which means a rather high quality 
for filtering and note, that it actually preserves the $95 \%$ of average power for OMA after its filtering thus, it means that the resulting SNR for demodulation of the OMA signal performs with only $3 \mathrm{~dB}$ losses by duplication of the resulting noise power, which is rather acceptable for SISO systems (and might be for MIMO as well!). In terms of Noise Immunity the application of general error-correcting codes could "compensate" those loses!

The latter sounds very opportunistic for transmission systems design, taking into account the drastic increase of its Spectrum Efficiency. Another interesting aspect is related to different channels conditions for OMA an NOMA: from Table 1 it follows, that applying the Filtering approach for SIC design, the SIR might be around 9-12 dB, i.e. almost similar to SNR and this states nothing else but, the possible numerical evaluation of the concept of sufficiently different channel conditions for users, mentioned above!

The last comment related to this issue is that the results for OMA Filtering are practically invariant to the attractor model used as reference for the filtering algorithm design (see Table 1).

Next, the results of filtering for NOMA signals in presence of AWGN noise are presented. In contrary to the experiments above (see figures 6 and 7), in this segment it is considered scenarios where the Interference to Noise Ratios is very small, which illustrates the possible accuracy for filtering users when, after extracting the OMA signal, NOMA interferences have to be filtered as well. Table 2 shows the results of those experiments in terms of the Interference to Noise ratio (INR) vs Normalized Mean Square Error (NMSE) with the corresponding Q values.

\begin{tabular}{|l|c|c|}
\hline INR & \multicolumn{1}{|c|}{$-3 \mathrm{~dB}$} & $-10 \mathrm{~dB}$ \\
\hline EKF Rossler X $\mathrm{Q}=0.1252$ & \\
\hline $1 \mathrm{MM}$ & 0.0112 & 0.0535 \\
\hline $2 \mathrm{MM}$ & 0.0108 & 0.0485 \\
\hline EKF Chua X $\mathrm{Q}=0.2$ & \\
\hline $1 \mathrm{MM}$ & 0.0154 & 0.0685 \\
\hline $2 \mathrm{MM}$ & 0.0143 & 0.0671 \\
\hline
\end{tabular}

Table 2. NMSE results for experiment with NOMA

Once more, it can be seen, that actually the proposed SIC filtering algorithms (see figures 1 and 2 ) are strictly working not as a decoder, but as a classifier for OMA and NOMA signals and when the OMA signal is well filtered (identified), then the multiuser interference separation or classification might be evaluated by the probability of classification error $P_{c l}$. The latter obviously affects the final error probability of the demodulation for all users $\left(P_{\text {err }}\right)$.

One of the possible ways of theoretically evaluating $P_{c l}$ for multi-user's classification (or for OMA-multiuser classification as well) is applying the so-called DGL, or Chernoff upper bound in the way [23]:

$$
P_{c l} \leq 2 m(m-1)^{2} \exp \left\{-n \frac{\Delta^{2}}{2}\right\},
$$

where $\Delta$ is the minimum Kullback-Leibler distance (see also (10)) between "closest" Gaussian distributions of the multiusers, $n$ is the number of samples and $m$ is the number of multiusers. For complete OMA-multiusers classification the total number of "users" is $m$ ' $=m+1$.

If $\forall P_{e r r}, P_{c l}<<1$ then it is easy to show that the total demodulation error $P$ for each signal is:

$$
P \approx P_{\text {err }}+P_{c l}
$$

and if practically $P_{c l} \geq P_{e r r}$, then, approximately $P_{c l}<P<$ $2 P_{c l}$, i.e. it depends mainly on the errors of the multiuser classification. So, from (9) it follows that minimization of $P_{c l}$ is crucial for the noise immunity of the NOMA transmission.

Note, that it is easy to illustrate, that for the Filtering SIC design the just above comment may not apply: assume that the Kullback-Leibler distance (metric for evaluating a significant difference for this scenario!) has the same order as SIR above, i.e. around " 4 " and $m$ is around 50, then it is simple to calculate, that the classification error from (8), even as an upper bound, is much less than the usually considered BER $\left(P_{\text {err }}\right)$ around $10^{-4}-10^{-6}$., i.e. it is negligible!.

In this regard the important question for implementation issues of the Filtering variant of SIC design arises: which signal has to be filtered first: OMA or multiusers? In the material presented above it was stated that OMA signal is filtered first, but in general this is an open question. It must be thoroughly investigated in the future, meanwhile some recent simulations show that filtering NOMA interferences first in order to "clean" the OMA signal (before its filtering) might be a rather complex issue, but it can give significant benefits in terms of reducing the NMSE for the OMA signal decoding.

\section{Multiuser Classification By Means OF "M" SEQUENTIAL HyPOTHESES TESTING AlgORITHMS}

Due to the sequential character of the multiuser decoding with SIC algorithms for CR-NOMA, it seems reasonable to consider a decoding strategy with a similar character, i.e. sequential for " $m$ " hypotheses testing.

Note that the main goal of this Section is exactly to attract attention to this approach for SIC design. The advantages of these algorithms for binary testing are well known long ago from A. Wald [24], but the results of their generalizations for the case of $m$ hypotheses testing are mainly addressed to its tiny mathematical problems [23][28].

For practical implementation, their solutions are rather complex except in few special cases. Other options (socalled "ad-hoc") so far, are based on heuristic extensions of the binary test for the case of $m$ hypotheses.

In this regard it was found reasonable to invoke the results from [28] together with the approach stated in [24] 
where the asymptotic approximations for the optimum Bayesian algorithms for hypothesis testing are presented for the case of small probabilities of decoding errors and an expected rather long testing time, particularly for low SNR cases. Both those assumptions seem realistic for SIC design in Doubly Selective channels, applying the Gaussian statistics as well.

It is pertinent to remind here that for efficient NOMA transmission, as it was commented above, sufficiently different channel conditions for the users are necessary, (see above). However, even if channel conditions for OMA and multi-users are very different, energetically between multi-users the differences might not be so drastic, i.e. hypotheses for multiusers only in this sense might be "close", and therefore the testing time might be large! So, application of m-ary sequential analysis for such kind of scenarios might be rather opportunistic!

The extractions from the material of [24], [28] for the asymptotic characteristics of the algorithm are as following:

$$
\begin{aligned}
& \left\langle N_{k}\right\rangle=-\frac{\log A_{k}}{\min _{j: j \neq k} D\left(f_{k}, f_{j}\right)} \\
& \alpha_{k}=\pi_{k} A_{k} \gamma_{k} \quad \alpha=\sum_{k=0}^{m-1} \pi_{k} A_{k} \gamma_{k}
\end{aligned}
$$

where $D(f, g)=\int f(x) \log \frac{f(x)}{g(x)} d x$ is the Kullback-Liebler distance between the PDFs $f(x)$ and $g(x) ;\left\langle N_{A}\right\rangle=\overline{N_{A}}$ is the average value of the first sample $n \geq 1$ when the decision for the hypotheses is taken; $\left\{\pi_{k}\right\}$ a priori probabilities of hypotheses $(k=\overline{1, m}) ;\left\{\gamma_{k}\right\}$ and $\left\{A_{k}\right\}$ are explained in the following.

In the usual case when the value of the error of the false alarm for probability for testing - $\alpha$ is a priori predefined, from (10) it follows:

$$
c=\frac{\alpha}{\sum_{k=0}^{m-1} \frac{\pi_{\kappa}}{\delta_{k}}}, \quad \delta_{k}=\min _{j: j \neq k} D\left(f_{k}, f_{j}\right), \quad A_{k}=\frac{c}{\delta_{k} \gamma_{k}}
$$

where $0 \leq \gamma_{k} \leq 1$ is tabulated at table 3.1 in [24] (see also [28]).

One has to notice, that for SIC design, applying the classification paradigm for multiusers classification, the predefined probability of the false alarm is nothing else, but a classification error, being usually assumed as a very low value much less than BER, the main interest for such kind of hypothesis testing presents a gain on the average time of analysis, i.e. how fast this testing is?

The technical gain of any sequential algorithm strongly depends on the thresholds choice which defines the decision regions [24], [28]; the data processing algorithm can be coherent (incoherent), energy detection, etc. and they are well known [24].

For the concrete case of Gaussian channels for all multiusers, where the decoding (classification) problem for multiusers depends on their SNR, QoS, amplitudes, covariations, average powers, etc. the formal scenario is as following.

Let $k=\overline{0, m-1}$ for hypothesis testing $\left\{H_{k}\right\}$ and $x_{1}, x_{2}, \ldots$ is a sequence of independent Gaussian random variables with means $\left\{\theta_{k}\right\}$ and variances $\sigma_{k}^{2}$, which are known a priori (see also [1], [3]). If $\left\{x_{k}\right\}$ are multiuser amplitudes, the data processing algorithm is obvious: $\sum_{i=0}^{n} x_{i}$, and the results have to be compared with the asymptotic thresholds for each " $k$ " as follows [28]:

$$
\begin{gathered}
\frac{n\left(\theta_{k}+\theta_{k+1}\right)}{2}+\frac{\log A_{k}}{\theta_{k+1}-\theta_{k}} \\
\frac{n\left(\theta_{k}+\theta_{k+1}\right)}{2}-\frac{\log A_{k+1}}{\theta_{k+1}-\theta_{k}}
\end{gathered}
$$

where $k=\overline{0, m-1}$.

It is interesting to express here an impressive issue: these asymptotic algorithms correspond exactly to the set of simple binary algorithms: $H_{0}$ vs $H_{1} ; H_{1}$ vs $H_{2}$ and so forth. This coincides totally with the intuitive processing of the $m$ tests as a straightforward generalization of the binary sequential test (called "ad-hoc", see the references in [26][28]) that was known long ago and shown at [28] to be asymptotically optimum!

Thus, for the Gaussian case the $m$ decoding asymptotic algorithm is nothing else but a "block" of cells each for the sequential testing of different binary hypotheses for the multiusers.

The examples of numerical results, for this testing can be found at [28], which, for sure, is not reasonable to reproduce here, show that for such kind of algorithms the number of samples (time of analysis) can be reduced 2 or 3 times compared to an optimum algorithm with fixed testing time, so they are relatively fast! The latter follows exactly from the convergence of the $m$ testing to the $(m-1)$ set of "binary" algorithms well known several decades ago and proofed as fast ones, presented first by A. Wald. Therefore, those algorithms are essential for SIC design for Doubly Selective channels.

To conclude this section, it is worth mentioning that due to the asymptotic character of the proposed algorithms, one has to notice that they might demonstrate robust features to the multi-user statistics as well; the latter requires further investigation.

\section{CONCLUding REMARKS AND FUture WORK PROPOSALS}

The material presented above is devoted to two original options for the SIC algorithm design for the power-domain CR-NOMA information transmission over Doubly 
Selective Channels. This type of channels take place at $5 \mathrm{G}$ and beyond for wireless information transmission from HSV terminals, but not only from them!

It was shown earlier [13]-[16] that it is rather "hard" to obtain high values for characteristics such as noise immunity and spectral efficiency for Doubly Selective channels due to numerous impairments and distortions that take place there. Application of the power-domain NOMA to improve the spectrum efficiency in those channels requires additional efforts in SIC processing to reduce the influence of the multiuser interferences.

For application in SISO channels, two possible approaches have been proposed for multi-user interferences mitigation through SIC, namely a Chaos-based filtering approach and an approach based on the $m$ sequential hypotheses testing. Both of them show several attractive features for application over Doubly Selective channels such as high fidelity for multiuser decoding, possible robustness and reduced decision time, which allows to recommend them not only for Doubly Selective channels, but also for the "conventional" channels as well, besides the large number of already reported algorithms for them (see [1]-[12] and references therein).

Meanwhile, one has to notice that the complete conclusions regarding efficiency of the proposed approach for SIC design for Doubly Selective channels might appear later, when the number of such proposals starts to be consistent and more material for comparative analysis will be available.

At the same time, it is worth noticing the existence of several unsolved problems for SIC design, particularly for Doubly Selective channels. Among others one can cite the following:

- The methods proposed here need to be exhaustively compared against a broad range of impairing features which exist in Doubly Selective channels.

- Generalizations of CR-NOMA for MIMO Doubly Selective channels and Massive MIMO. It seems that, although the SIC can be considered as a "pre-processor block" before the main receiving "unit" (the latter is already well known [13]-[16]), for applications in SISO and MIMO over Doubly Selective channels the before mentioned algorithms might require some modifications.

- The power-domain NOMA paradigm (for example, CRNOMA) has to be generalized by means of invariant methods for incoherent modulation and demodulation (see [16]).

-It is worth to mention for the future work the possible applications as well of the user's grouping to-gether with the proposed trend for SIC design at NOMA transmission.

Sure, it is impossible to consider all the problems in the framework of one single paper and they might be a topic for further research.

\section{ACKNOWLEDGMENT}

Author is happy to express his deep gratitude to Dr.
Fernando Ramos for his help in developing the simulations presented in this material and to Dr. Beatriz Rodriguez for her help in the search and analysis of the related references.

\section{REFERENCES}

[1] Z. Ding, R. Schober, H. Vincent Poor, "unveiling the importance of SIC in NOMA systems", Parts I and II, IEEE Communication Letters vol. 24, no. 11, pp. 2373-2382, Nov. 2020.

[2] F.-Y. Tian, X.-M. Chen, "Multiple antenna techniques in nonorthogonal multiple access: a Review", Frontiers of Information Technology and Electronic Engineering, vol. 20, no. 12, pp. 16651697, 2019.

[3] N. Iswarja, L.S. Jayashree, "A survey of successive interference cancellation schemes in in non-orthogonal multiple access for future radio access", Wireless Personal Communications, Published on line, April 26th 2021.

[4] H-N. Nguyen, S-P. Le, C-B Le, et al., "Cognitive Radio Assisted Non-Orthogonal Multiple Access: Outage Performance", 42nd Int. Conference on Telecommunications and Signal Processing (TSP), Budapest, Hungary, 2019.

[5] X. Liu, Y. Wang, S. Liu, J. Meng, "Spectrum Resource Optimization for NOMA-Based Cognitive Radio in 5G Communications". IEEE Access vol. 6, pp. 24904-24911, 2018. doi: 10.1109/access. 2018.2828801 .

[6] T. Wang, L. Shi, K. Cai, L. Tian, S. Zhang, "Non-Coherent NOMA With Massive MIMO", IEEE Wireless Communications Letters vol. 9, no. 2, pp. 134-138, Feb. 2020.

[7] T. Xu and I. Darwazeh, "Non-Orthogonal Frequency Division Multiple Access", IEEE 91st Vehicular Technology Conference (VTC2020-Spring), Antwerp, Belgium 2020.

[8] K. S. Ali, E. Hossain, M. J. Hossain, "Partial Non-Orthogonal Multiple Access (NOMA) in Downlink Poisson Networks", IEEE Transactions on Wireless Communications, vol. 19, no. 11, pp. 76377652, Nov. 2020.

[9] K. Saito, A. Benjebbour, A. Harada, Y. Kishiyama, T. Nakamura, "Link-Level Performance of Downlink NOMA with SIC Receiver Considering Error Vector Magnitude", IEEE 81st Vehicular Technology Conference (VTC-Spring), Glasgow, United Kingdom, 2015.

[10] F. Y. Tian, X. M. Chen, "Multiple-antenna techniques in nonorthogonal multiple access: a review", Frontiers of Information Technology and Electronic Engineering, vol.20, no. 12, pp.16651697, 2019.

[11] A. Ruegg , A. Tarable, "Iterative SIC receiver scheme for nonorthogonally superimposed signals on top of OFDMA", IEEE 21st Annual International Symposium on Personal, Indoor and Mobile Radio Communications (PIMRC), Istanbul, Turkey, 2010.

[12] R. Alberto, F. A. Monteiro, "Downlink MIMO-NOMA With and Without CSI: A Short Survey and Comparison", 12th International Symposium on Commuication Systems, Networks and Digital Signal Processing (CSNDSP), Porto, Portugal, 2020.

[13] F. Peña-Campos, R. Parra-Michel and V. Kontorovich, "A low complexity multicarrier system over doubly-selective channels using virtual-trajectory receiver", IEEE Transactions on Wireless Communications, vol. 15, no. 8, pp. 5206-5217, Apr. 2016.

[14] F. Peña-Campos, R. Parra-Michel and V. Kontorovich, "Incoherent DPSK diversity reception in Doubly-Selective channels based on virtual trajectories", IEEE Transactions on Vehicular Technology, vol. 67, no. 8, pp. 7744-7748, Mar. 2018.

[15] F. Peña-Campos, R. Parra-Michel and V. Kontorovich, "MIMO multicarrier transmission over Doubly Selective channels with virtual trajectories receiver", IEEE Transactions on Vehicular Technology, vol. 68, no. 10, pp. 9930-9338, Aug. 2019.

[16] G. Ramirez-Arredondo, F. Peña-Campos, R. Parra-Michel and V. Kontorovich, "Invariant incoherent MIMO reception over doublyselective channels", IEEE Access, vol. 9, pp. 67776-67785, May. 2021.

[17] V. Kontorovich, Z. Lovtchikova, "Nonlinear Filtering of Chaos for real Time applications", chapter 3 in Selected Topics in nonlinear Dynamics and Theoretical Electrical Engineering, edited by K. Kyamakya, W. A. Halang, et al.,. Springer-Verlag, 2013.

[18] H. Van Trees, Detection, Estimation and Modulation Theory, John Wiley \& Sons, 2001. 
[19] A. Jazwinski, Stochastic Processing and Filtering Theory, N.Y. Academic, 1970.

[20] V. Kontorovich and F. Ramos-Alarcon, "Ubiquitous filtering for nonlinear problems", Chapter 5 in Research Advances in Chaos Theory, Ed. Intech Ltd. 2019.

[21] V. Kontorovich, Z. Lovtchikova, "Cumulant Analysis of Strange Attractors: Theory and Applications", in Recent Advances in NonLinear Dynamics and Synchronization, Springer-Verlag, edited by K. Kyamakya, W. A. Halang, et al., 2009, p.p. 77-115.

[22] D. Arditti, A. Alcocer, F. Ramos, V. Kontorovich. "Adaptive mitigation of platform-generated radio-frequency interference", Patents EP2838205A3, 2014 and CN104378128B, EPO, 2018.

[23] L. Devroye, L. Gyorfi and G. Lugosi, "A note on robust hypothesis testing", IEEE Transaction on Information Theory, vol. 48, no. 7, pp. 2111-2114, Aug. 2002.

[24] M. Woodroofe, "Nonlinear renewal theory in sequential analysis", SIAM, 1982.

[25] A. Malek, S. Katariya, Y. Chow and M.Ghavamzadeh, "Sequential multiple hypothesis testing with type I error control", Proceedings of the 20th International Conference on Artificial Intelligence and Statistics, USA, 2017, v. 54, pp. 1468-1476.

[26] A. Novikov, "Optimal sequential multiple hypothesis tests", Kybernetika, vol. 45, no. 2, pp. 309-330, 2009.

[27] H. Afser, "Statistical classification via robust hypothesis testing", arXiv: 2106.04824v1 [cs.IT], jun 9th, 2021.

[28] C. W. Baum and V. V. Veeravalli, "A sequential procedure for multihypothesis testing", IEEE Transactions on Information Theory, vol. 40, no. 6, pp. 1994-2007, Nov. 1994.

Valeri Kontorovich was born in the former Soviet Union, now Russia . He received the M.S.E.E. (1963), Ph.D. in Telecommunications (1968) and Dr. Sc. (1987) in Telecommunications, all degrees from St. Petersburg State University of Telecommunications (St. Pt. UT) of Russia.

From 1964 to 1965 he was Technical Staff member of the State Radio Institute. In 1965, he joined the St. Pt. UT, where in 1976 he founded the Laboratory of Electromagnetic Compatibility (EMC lab). In 1984 this laboratory was transformed into Branch Lab of the Ministry of Telecommunications of the former USSR. Since 1988 he is full professor and was a head of department from 1992 to 1995 . Since 1993 he is full professor at the Research and Advanced Studies Center (CINVESTAVIPN) of the National Polytechnic Institute (IPN) in Mexico. He has published more than ten books in Russian, English and Spanish and more than 300 journal and conference papers. He holds more than 25 international patents. His present interests are advanced mobile radio communication systems, interference analysis, channel modeling, and communications theory in general.

Dr. Kontorovich is member of the Mexican Academy of Sciences, "Emeritus" member of the Mexican National Researchers System (SNI) and Life Fellow of the IEEE. 